\title{
4 PROGRAMA DE LUTA CONTA O ESTIGMA: RESULTADOS OBTIDOS NA FORMAÇÃO NOS PROFISSIONAIS DA SAÚDE MENTAL
}

\author{
| Francisco Javier Barrantes ${ }^{1}$; Carlos Violante ${ }^{2}$; Luís Graça ${ }^{3}$; Isabel Amorim ${ }^{4}$ |
}

\section{RESUMO}

Os utentes dos serviços de saúde mental sentem-se estigmatizados pelos profissionais. Analisam-se os resultados obtidos numa ação formativa, dirigida aos profissionais da Casa de Saúde São João de Deus-Barcelos. Construiu-se um estudo comparativo: 1\% Efetuou-se um diagnóstico de situação; 2\% avaliaram-se os resultados após intervenção. Utilizado o instrumento AQ-27, versão portuguesa. Não se observam diferenças significativas, à exceção do evitamento. As ações informativas devem integrar-se em processos interativos, não serem "informação sobre" mas sim "conhecimento de / junto de", havendo uma maior interatividade (colaboradores-utente).

\section{PALAVRAS-CHAVE: Estigma social; saúde mental; profissionais da saúde; formação continua}

\section{RESUMEN}

"Programa de lucha contra el estigma: resultados obtenidos en la formación dirigida a los profesionales de la Salud Mental”

Los clientes de los servicios de salud mental se sienten estigmatizados por los profesionales. Analizamos los resultados obtenidos en una formación dirigida a profesionales. Se elaboró un estudio comparativo: $1 \%$ Se efectuó un diagnóstico; $2 \%$ Se valoraron los resultados obtenidos después de esta intervención. Utilizado instrumento AQ-27, versión portuguesa. No se observan diferencias significativas, excepto en el factor Evitación. Las acciones informativas deben integrarse en procesos interactivos, no siendo sólo "acciones de información sobre" y sí "conocimiento de/junto de".

DESCRIPTORES: Estigma social; salud mental; profesionales de la salud; formación continua

\begin{abstract}
"Fight against Stigma Program: results obtained in the training of Mental Health Professionals"

The users of mental health services feel stigmatized by professionals. In this article are analysed the results obtained in a workshop/course, directed to the Institution professionals. A comparative study was elaborated, in two moments: $1 \%$, Diagnosis was made; $2 \%$ it was intended to evaluate the results after intervention. The instrument AQ-27, Portuguese version. No significant differences were observed, with the exception of avoidance. It is stated that workshops/courses should be integrated in interactive processes, not only "information about" but, rather "knowledge of / with who (employees-users).
\end{abstract}

KEYWORDS: Social stigma; mental health; health professionals; continuing education

Submetido em 15-12-2016

Aceite em 20-05-2017 


\section{INTRODUÇÃO}

Segundo a conceptualização clássica (Goffman, 1963, p.6) o estigma é um atributo profundamente depreciativo que transforma o portador, passando de ser uma pessoa normal para outra desonrada e menosprezada ou sendo simplesmente estranha às pessoas que a identificam. Hoje em dia, de acordo com alguns autores (Corrigan, 2004a; Lopez et al., 2004), é estabelecida a diferença entre "estigma público e privado". "Estigma público" é o fenómeno característico de grandes grupos sociais que apoiam determinado estereótipo e agem em conformidade contra um grupo estigmatizado (ex. pessoas com doença mental); "Estigma privado ou Auto-Estigma" é definido como a perda de auto-estima e auto-eficácia que decorre de a pessoa interiorizar o Estigma Público. Tanto o Estigma Público, como o Privado compõem-se de traços cognitivos - comportamentais. Estes traços cognitivos ou atitudes diferenciam-se entre estereótipos (crenças negativas sobre a pessoa com perturbação mental) e preconceitos (reações emocionais que resultam da transferência dos estereótipos). A perigosidade, a imprevisibilidade do comportamento, a incompetência do indivíduo, a infantilidade, a culpabilidade e o contágio são os estereótipos negativos associados às pessoas com doença mental e que resultam em reações emocionais ou preconceitos, também negativas, nomeadamente o medo, a raiva, a pena e a repugnância. Estas atitudes originam comportamentos discriminatórios tais como a coação, o evitamento e a pouca consideração às necessidades apresentadas por parte das pessoas com experiência em doença mental.

A estigmatização inicia-se com a distinção, etiquetado ou "labeling" e identificação de alguma diferença que afeta a pessoa com doença mental. Posteriormente haverá uma associação, destas pessoas diagnosticadas, com etiquetas desagradáveis condicionadas por crenças culturais prevalentes. Segue-se uma consideração, para com estes indivíduos, como sendo um grupo diferente, estabelecendo-se uma divisão entre "eles" e "nós". Existindo estas divisões, aparecem repercussões emocionais que afetam, quem estigmatiza (medo, ansiedade, irritação e compaixão) e quem fica estigmatizado (medo, ansiedade e vergonha). O resultado deste processo é que os indivíduos estigmatizados podem perder o seu status dentro dos grupos sociais onde estão inseridos (não serem aceites como amigos, vizinhos, empregados, maridos, esposas, colegas de estudo...).
Esta situação restringe os seus direitos e oportunidades, por motivo destes preconceitos funcionarem como uma barreira no acesso à vida social plena e aos serviços de todo tipo que possam necessitar, ficando desta forma, dependentes dos "outros".

Segundo a literatura científica (Angermeyer, 2006; Arvanti et al., 2008; Corrigan e Watson, 2002) os utentes dos serviços de saúde mental sentem-se estigmatizados também pelos profissionais, por diversos motivos: a falta de interesse manifestado pelos profissionais em relação ao utente e à sua história de vida; sentimento generalizado de um tratamento farmacológico (dosagem e tipo de drogas) estandardizado; perceção de diagnósticos associados a prognósticos negativos; informação insuficiente em relação ao tratamento e opções de acompanhamento terapêutico na comunidade. Um outro aspeto que poderá contribuir para esta estigmatização e discriminação são os efeitos secundários da medicação. Considerando o novo paradigma de abordagem da doença mental torna-se de fundamental importância romper com este ciclo que se constitui como um grande obstáculo à participação social e ao "recovery" destas pessoas, pelo facto de restringir as oportunidades a que elas têm direito.

Neste contexto, percebendo, quer as dificuldades sentidas pelos profissionais da Casa de Saúde S. João de Deus (CSSJD) - Barcelos (estabelecimento do Instituto S. João de Deus, cujo âmbito de intervenção é a doença mental e cujo objetivo se centra na filosofia hospitaleira da Ordem de S. João de Deus), quer as dificuldades ao nível da população da área onde se encontra localizada a Instituição, surgiu em 2013, uma proposta de Projeto de Intervenção, em parceria com vários agentes locais, na e para a comunidade, que se denominou "Luta contra o Estigma". Este projeto trabalhou, através das suas diferentes atividades, a identificação e consequente intervenção para eliminação ou diminuição dos estereótipos que ainda pudessem existir, utilizando, para isso, os princípios referidos na literatura científica para a luta contra o estigma, nomeadamente: Mobilização social e protesto, informação e educação referentes à doença mental e o contato e interação social com pessoas com experiência em doença mental, apresentando como objetivos principais:

Diminuir o impacto do estigma da doença mental na comunidade, fomentando o debate público acerca da doença mental e do estigma associado; 
Desmistificar a doença mental, difundindo imagens positivas e possibilitar o empowerment e recovery dos indivíduos com doença mental. No âmbito deste projeto, foi levada a cabo uma atividade de formação para os colaboradores da Instituição cujo objetivo fundamental seria fomentar a reflexão e a mudança de atitudes acerca da doença mental nos próprios profissionais de saúde mental.

Com vista a podermos proceder à avaliação da atividade desenvolvida com os profissionais da Instituição, num primeiro momento efetuamos uma avaliação inicial relativamente ao estigma face à doença mental, e após a intervenção procedemos a nova avaliação, entre os profissionais que participaram na formação. Com este trabalho pretendemos apresentar alguns dos resultados obtidos acerca das perceções tidas pelos profissionais antes e após a ação de sensibilização referida.

O objetivo do estudo é avaliar o contributo de uma intervenção junto dos profissionais da CSSJD - Barcelos relativamente às atitudes perante o estigma na doença mental.

\section{METODOLOGIA}

Face ao objetivo, construiu-se um estudo comparativo, em dois momentos, ainda que não haja completa coincidência entre os grupos. No primeiro momento efetuou-se um diagnóstico de situação e no segundo momento pretendeu-se avaliar resultados após a intervenção.

A população é constituída pelos profissionais que em todo ou em parte exercem funções na CSSJD - Barcelos. A amostra ficou constituída pelos profissionais que se disponibilizaram a colaborar no estudo e com informação suficiente nos questionários. Assim ficou constituída no primeiro momento por 46 profissionais, e no segundo momento por 27 profissionais. $\mathrm{O}$ presente estudo sustenta-se na hipótese que a intervenção desenvolvida, contribuiu para a diminuição do estigma relativamente à doença mental. A intervenção consistiu numa ação de formação de duas horas com o tema "Estigma face à doença mental - Atitudes dos profissionais de saúde", que teve como objetivos contextualizar o estigma face à doença mental e fomentar a reflexão e a mudança de atitudes nos profissionais de saúde. Foram utilizadas como técnicas pedagógicas a exposição de conteúdos e a discussão crítica com recurso a exemplos práticos.
Os conteúdos programáticos definidos foram um enquadramento teórico da temática, onde foram definidos conceitos como "estereótipo", "preconceito" e "discriminação"; foram contextualizados termos como "estigma público" e "estigma privado" fazendo paralelo com fenómeno social do estigma e com o estigma sentido pelo próprio; foi efetuada uma reflexão acerca das atitudes estigmatizantes e discutidos os motivos de tais perceções; foram apresentados os resultados iniciais da colheita de dados desenvolvida na Instituição referente a esta temática, refletindo e discutindo alguns indicadores de possíveis atitudes estigmatizantes e por fim foram expostas algumas medidas que possam garantir uma mudança de atitude, por parte dos profissionais. Esta intervenção constitui-se como variável Independente.

Face ao objetivo do estudo a nossa variável dependente foi o estigma face à doença mental, avaliado através do "The Attribution Questionnaire 27" de Corrigan (2004) na versão portuguesa de Sousa, Queirós, Marques, Rocha e Fernandes (2008). O Questionário de Atribuição AQ-27 (“The Attribution Questionnaire 27”) de Corrigan (2004) é utilizado para medir os elementos do preconceito relativo às pessoas com doença mental. O AQ-27 tem sido utilizado em alguns estudos e tem demonstrado ser um questionário sensível para medir as várias atitudes, assim como outras reações prejudiciais sofridas pelas pessoas com uma doença mental. Conforme Corrigan et al. (2004) o questionário é constituído por 27 itens divididos em nove fatores/domínios (três itens por fator/domínio): Responsabilidade, Pena, Irritação, Perigosidade, Medo, Ajuda, Coação, Segregação e Evitamento. Cada item é operacionalizado numa escala ordinal com nove atributos. Quanto maior a pontuação em cada um destes fatores/domínios, mais estigma está representado no sujeito. Os três itens do domínio Evitamento (7, 16 e 26) têm uma cotação invertida. Nesta investigação o questionário revelou boa consistência interna (Alfa de Cronbach - 0,817). A versão portuguesa do questionário é acompanhada por quatro histórias alternativas ou vinhetas. Todas se centram em histórias de uma pessoa com diagnóstico de esquizofrenia, embora cada uma delas apresente diferentes condições: sem perigo, perigo, perigo sem controlo de causa e perigo com controlo de causa. Neste estudo foi apresentado o José com a condição de perigo, visto que este é um dos estereótipos comumente partilhado por muitos dos profissionais da saúde mental e poderá representar situações que podem ocorrer nas unidades/serviços em estudo. 
Tendo por referência o objetivo do presente trabalho, recorreu-se a técnicas estatísticas descritivas. Posteriormente procedeu-se à análise da homogeneidade entre os grupos, uma vez que os dados não permitiam o emparelhamento, utilizando-se testes para amostras independentes. Assim, recorreu-se ao teste de independência de qui-quadrado, ao teste U-MannWhitney e ao teste $t$ student. Para a avaliação da normalidade de distribuição utilizou-se o teste de Shapiro-Wilk. Considerou-se um nível de significância de $5 \%$ e o software utilizado foi o Statistical Package for the Social Sciences- versão 21. A investigação foi conduzida com o princípio do respeito pela autonomia e dignidade da pessoa, pelo que se respeitou o direito à confidencialidade, anonimato e privacidade.

\section{RESULTADOS}

No que se refere às características sociodemográficas, quanto ao sexo predomina o masculino (63,0\%). As idades variam entre os 20 e os 60 anos, com média de $40,97 \pm 12,04$ anos e mediana 42 anos. Quando consideramos os grupos etários, o mais representado é o com 50 anos e mais $(31,5 \%)$ apresentando os restantes grupos etários distribuição idêntica. No que se refere à escolaridade a maioria tem o ensino básico $(45,2 \%)$, seguido dos com Licenciatura $(37,0 \%)$, sendo que 2,7\% tem como habilitações o mestrado. Quanto ao estado civil são maioritariamente casados ou vivem em união de facto $(56,2 \%)$, sendo o grupo menos representado o dos divorciados (9,6\%), sendo que $2,7 \%$ não responderam. Relativamente à idade não se observou normalidade de distribuição (ShapiroWilk: momento 1 - 0,920; gl 46; sig 0,004; momento 2 - 0,897; gl 27; sig 0,011), no entanto também não se observou assimetria ou kurtose severas, pelo que se utilizou o teste de $\mathrm{t}$ para amostras independentes. Em nenhuma das variáveis sociodemográficas se observou diferenças estatisticamente significativas entre os momentos.

As características profissionais referem-se à ocupação, onde predominam os empregados auxiliares $(63,0 \%)$, seguidos dos enfermeiros com $37,0 \%$, apresentando os restantes grupos profissionais distribuições muito menores (de $1,4 \%$ a 5,5\%). No que se refere ao local de trabalho a maioria exerce funções exclusivamente em unidades de internamento $(79,5 \%)$ ou unidades de internamento e outras unidades (5,5\%), trabalhando os restantes $15,1 \%$ em unidades sem internamento.
O desempenho dos profissionais faz-se maioritariamente junto de doentes com patologia crónica $(60,3 \%)$, seguido dos que prestam serviço junto de doentes crónicos e agudos (24,7\%).

Para efeitos da análise da homogeneidade entre os momentos optámos por agrupar a ocupação em dois grupos, um em que se incluem os profissionais de saúde com formação diferenciada em saúde e outro com os restantes profissionais sem formação específica em saúde. Relativamente ao local de trabalho constituíram-se dois grupos: um em que os profissionais exercem atividade no todo ou em parte no internamento e outro em que não exercem atividades em internamento. Entre os momentos observam-se diferenças quanto ao local de trabalho, não se observando nas restantes variáveis. Da análise de resíduos ajustados standardizados observam-se mais profissionais com atividade em unidades de internamento que o esperado, enquanto no segundo momento se observa maior proporção de profissionais com atividade em serviços sem internamento que o esperado. Constata-se que no primeiro momento não havia nenhum profissional cuja atividade fosse só em unidades sem internamento.

Quadro 1 - Mediadas de estatística descritiva das dimensões do "Attribution Questionnaire 27"

\begin{tabular}{|l|c|c|c|c|}
\hline & Min-Max. & Média \pm D.P & Mediana & P25-P75 \\
\hline $\begin{array}{l}\text { Responsabi- } \\
\text { lidade }\end{array}$ & $3-18$ & $8,39 \pm 3,70$ & 8,0 & $5,0-11,0$ \\
\hline Pena & $3-27$ & $17,89 \pm 5,84$ & 18,0 & $13,0-23,0$ \\
\hline Irritação & $3-27$ & $8,73 \pm 4,19$ & 8,0 & $5,0-11,0$ \\
\hline Perigosidade & $5-26$ & $14,23 \pm 5,57$ & 14,0 & $10,5-18,0$ \\
\hline Medo & $3-25$ & $11,30 \pm 6,14$ & 9,0 & $6,0-17,0$ \\
\hline Ajuda & $7-27$ & $22,03 \pm 4,44$ & 22,0 & $19,0-26,0$ \\
\hline Coação & $8-27$ & $18,89 \pm 3,91$ & 19,5 & $17,0-$ \\
\hline Segregação & $4-26$ & $15,32 \pm 5,17$ & 15,0 & $12,0-19,0$ \\
\hline Evitamento & $3-27$ & $15,82 \pm 5,65$ & 16,0 & $13,0-19,0$ \\
\hline
\end{tabular}

As dimensões do estigma, foram avaliadas através do AQ-27. Relativamente aos domínios (quadro $\mathrm{n}^{\circ} 1$ ) é na responsabilidade e na irritação que se observam as médias mais baixas $(8,39 \pm 3,70$ e $8,73 \pm 4,19$, respetivamente), apresentando medidas de posição idênticas (P25 e P75). A perigosidade, a Segregação e o Evitamento apresentam médias semelhantes. A média mais elevada observa-se na ajuda $(22,03 \pm 4,44)$, que apresenta também uma das menores amplitudes (727). 
De uma forma geral observa-se normalidade de distribuição e nas situações em que não se observa, à exceção da Irritação no primeiro momento, não se observa assimetria ou kurtose severas. Assim para a análise das diferenças entre os dois momentos, optouse pelo teste de $t$ para amostras independentes, à exceção da irritação, que se recorreu ao teste U-MannWhitney.

Quadro 2 - Análise das diferenças entre os momentos quanto ao estigma

\begin{tabular}{|l|l|c|c|}
\hline Variável & Valor de teste & $\mathrm{gl}$ & Sig \\
\hline Responsabilidade $^{*}$ & $-0,646$ & 69 & 0,520 \\
\hline Pena $^{*}$ & $-0,785$ & 71 & 0,435 \\
\hline Irritação $^{* *}$ & 493,000 & - & 0,142 \\
\hline Perigosidade* & 1,281 & 71 & 0,204 \\
\hline Medo $^{*}$ & 0,952 & 71 & 0,344 \\
\hline Ajuda $^{*}$ & $-1,615$ & 71 & 0,111 \\
\hline Coação* $^{*}$ & 0,444 & 70 & 0,658 \\
\hline Segregação & 1,586 & 71 & 0,117 \\
\hline Evitamento $^{*}$ & 2,791 & 71 & 0,007 \\
\hline
\end{tabular}

${ }^{*}$ teste de t para amostras independentes; ${ }^{*}$ teste U Mann-Whitney

Entre os momentos não se observam diferenças significativas, à exceção do evitamento, em que os valores do primeiro momento são significativamente mais elevados que no segundo $(17,17 \pm 5,42$ vs $13,52 \pm 5,37)$.

\section{DISCUSSÃO}

Está demonstrado que a experiência pessoal com os utentes com perturbação psiquiátrica reduz as valorações negativas atribuídas aos mesmos, porque diminui, entre outras, a perceção de perigosidade e imprevisibilidade associadas à perturbação. Na bibliografia revisada é referido que o medo e a perigosidade são duas atitudes geradoras de comportamentos discriminatórios e que podem, consequentemente, condicionar também os outros domínios estudados na escala. De referir que esta intervenção pretendia, impedir a perpetuação, junto dos colaboradores, do processo de estigmatização, como forma de eliminar os preconceitos que pudessem ainda existir.

Os resultados revelaram valores superiores, no primeiro momento do estudo, no domínio de Evitamento. A interpretação destes resultados tem de ser tomada com cautela, visto que não existem até ao momento, muitos estudos específicos que abordem o estigma dos profissionais da Saúde junto de pessoas com uma doença mental relacionado com este domínio específico.
Contudo, os dados obtidos parecem demostrar que não será o tempo de serviço na saúde mental que determinará o resultado obtido.

Porém, as habilitações académicas parecem ser um dos fatores que nos pode ajudar a explicá-los dado que a amostra mais significativa faz referência aos colaboradores com ensino básico. Assim, o baixo nível de habilitações pode ser um dos aspetos que condiciona a formulação de atitudes mais positivas perante as pessoas doentes: Havendo menos conhecimentos em relação às doenças mentais, existem mais dificuldades em filtrar adequadamente as informações erróneas que são transmitidas através dos meios de comunicação social, por exemplo, que apresentam estes utentes, como sendo pessoas não capacitadas para trabalhar ou mesmo para serem vizinhos. Evidenciamos aqui que, o facto de estes colaboradores terem participado na atividade formativa, terá tido efeitos positivos no que concerne à diminuição dos estereótipos e mais especificamente no domínio do Evitamento.

Outro aspeto relevante é o facto de 31,5\% da amostra, que corresponde em grande parte aos empregados auxiliares da Instituição, terem uma idade superior a 50 anos, tendo a sua residência nas imediações do seu local de trabalho. Estes dois fatores poderão ter influenciado nos resultados apresentados. Os hospitais psiquiátricos têm sido, com frequência, construídos no campo, crescendo, nas zonas circundantes dos hospitais, lojas para os profissionais que trabalhavam nestas instituições e para os próprios utentes, tornando-se frequente e comum a tradição familiar de trabalhar como profissional de saúde no hospital. Assim, nas instituições mais antigas, várias gerações podiam ser empregadas desta forma, desenvolvendose uma cultura de cuidados com um cariz particularmente protetor. Os domínios de Pena e de Medo, que serão aqueles mais diretamente relacionados e influenciados com o cariz protetor, por exemplo, não tiveram grandes diferenças nos dois momentos do estudo, situação que explica o facto de estes estereótipos estarem, por enquanto, bastante cristalizados nestes colaboradores, apesar de terem participado na formação e conviverem, quase diariamente, com pessoas doentes. A tipologia de unidades em que se trabalha, segundo os diferentes estudos, pode influenciar a perpetuação dos estereótipos. Num estudo desenvolvido anteriormente (Barrantes, 2010) evidenciouse que as unidades de longo internamento seriam um fator importante de estigmatização, situação que não foi verificada neste novo estudo. 


\section{CONCLUSÃO}

Embora a formação por si só não garanta mudanças efetivas nas atitudes nem nos comportamentos dos colaboradores, como se evidenciou no estudo, é preciso dar informação correta segundo os objetivos, conteúdos e grupos profissionais, preferencialmente integrados nos sistemas habituais das tarefas/procedimentos a executar dentro da Instituição, evitando que este tipo de formações sejam um momento de, simplesmente, "dar a conhecer". Assim a informação deve integrarse em processos dinâmicos e interativos, não serem "informação sobre" mas sim "conhecimento de / junto de", havendo uma maior interatividade entre colaboradores e utentes em fase de recovery, favorecendo o contato e conhecimento do dia-a-dia das pessoas com estas incapacidades. Para além do referido, é preciso identificar, dentro da Instituição, quais as fontes que promovem o aparecimento ou continuidade destes estereótipos, apesar de referido que estas ideias são geralmente infundadas mas influenciadas por fontes que deverão ser devidamente reconhecidas, sem a qual dificilmente será possível mudar os possíveis preconceitos existentes. Saliente-se ainda que as notícias nos meios de comunicação social, na maior parte das vezes, quando relacionadas com as pessoas com incapacidade psicossocial, associam-nas a acontecimentos negativos que promovem estes mesmos estereótipos, sendo da responsabilidade dos colaboradores filtrar e discutir estas informações, como forma de não condicionar o seu trabalho diário. Também compete à Instituição dar a conhecer aspetos positivos do desempenho dos utentes. Desta forma, evidencia-se que, apesar de incapacidades, o utente tem capacidade para escolha, decisão e competências para desenvolver uma vida com qualidade.

\section{IMPLICAÇÕES PARA A PRÁTICA CLÍNICA}

São recomendadas algumas medidas que possibilitarão a mudança de atitudes, de forma a evitar o estigma: Incrementar a sensibilização perante o poder "estigmatizante" do diagnóstico clínico na saúde mental, o que inclui a necessidade de saber se certas informações têm de ser ou não transmitidas aos próprios utentes, impedindo a estigmatização do mesmo; Fomentar, dentro da Instituição, o empoderamento e empowerment dos utentes, como forma de diminuir o cariz protetor dos colaboradores; Envolver significativamente o utente e os seus familiares nos programas anti-estigma; Garantir uma correta atenção por parte dos serviços sócio-sanitários, baseada nos modelos do recovery.
É necessário planificar e manter a longo prazo os programas implementados com o objetivo de erradicar o estigma, combinados com campanhas de sensibilização focadas em reduzir a descriminação, através da reflexão sobre as atitudes e as conceções sobre a doença mental. Informar e educar nas escolas, nos centros de trabalho, na rede sócio - sanitária e nas empresas de comunicação social, são passos para suprimir os mitos, aceitar a doença mental e lograr a integração das pessoas que estão diagnosticadas, Defendendo-se igualmente o "contacto" com estas pessoas, como sendo este um dos fatores que mais influenciam na mudança de atitudes, em combinação com os anteriores.

\section{REFERÊNCIAS BIBLIOGRÁFICAS}

Angermeyer, M., \& Dietrich, S. (2006). Public beliefs about and attitude towards people with mental illness: A review of population studies. Acta Psychiatrica Scandinavica, 113, 163-179. doi:10.1111/j.16000447.2005.00699.x

Arvaniti, A., Samakouri, M., Kalamara, E., Bochtsou, V., Bikos, C., \& Livaditis, M. (2008). Health service staff's attitudes towards patients with mental illness. Social Psychiatry and Psychiatric Epidemmiology, 44, 658665. doi: 10.1007/s00127-008-0481-3.

Barrantes, F. (2010) O estigma na esquizofrenia: Atitude dos profissionais da Saúde Mental. Tese de Mestrado, Universidade Católica Portuguesa, Portugal.

Corrigan, P. (2004a). Beat the stigma and discrimination! Four lessons for mental health advocates. [Em linha]. Disponível em: http://www.dmh.ca.gov/PEIStatewideProjects/docs/CorriganBeattheStigmaandDiscrimination.pdf. [Consultado em: 06/06/2016].

Corrigan, P., Markowitz, F., \& Watson, A. (2004). Structural levels of mental illness stigma and discrimination. Schizophrenia Bulletin, 30(3), 481-491.

Corrigan, P., \& Watson, A. (2002). Understanding the impact of stigma on people with mental illness. World Psychiatry, 1(1), 16-20.

Goffman, E. (1988). Estigma. Notas sobre a manipulação da identidade deteriorada. Rio de Janeiro: Guanabara.

López, M., Laviana, M., Fernández, L., López, A., Rodríguez, A., \& Aparício, A. (2008). La lucha contra el estigma y la discriminación en salud mental. Una estrategia compleja basada en la información disponible. Revista Asociación Española de Neuropsiquiatría ,28(101), 43-83. 